\title{
Migraine and risk of perioperative ischemic stroke and hospital readmission: hospital based registry study
}

\author{
(@) $(\Theta \Theta$ OPEN ACCESS
}

\author{
Fanny P Timm medical student ${ }^{1}$, Timothy T Houle associate professor ${ }^{1}$, Stephanie D Grabitz medical \\ student ${ }^{1}$, Anne-Louise Linn medical student ${ }^{12}$, Janne B Stokholm medical student ${ }^{12}$, Katharina \\ Eikermann-Haerter assistant professor ${ }^{3}$, Ala Nozari associate professor ${ }^{1}$, Tobias Kurth professor ${ }^{4}$, \\ Matthias Eikermann clinical director ${ }^{1}$
}

${ }^{1}$ Department of Anesthesia, Critical Care, and Pain Medicine, Massachusetts General Hospital and Harvard Medical School, 55 Fruit Street, Boston, MA 02114, USA; ${ }^{2}$ Department of Anesthesiology, University of Copenhagen, Herlev Hospital, Copenhagen, Denmark; ${ }^{3}$ Department of Radiology, Massachusetts General Hospital and Harvard Medical School, Boston, MA, USA; ${ }^{4}$ Institute of Public Health, Charité - Universitätsmedizin Berlin, Germany

\begin{abstract}
Objective To evaluate whether patients with migraine are at increased risk of perioperative ischemic stroke and whether this may lead to an increased hospital readmission rate.

Design Prospective hospital registry study.

Setting Massachusetts General Hospital and two satellite campuses between January 2007 and August 2014

Participants 124558 surgical patients (mean age 52.6 years; $54.5 \%$ women).

Main outcome measures The primary outcome was perioperative ischemic stroke occurring within 30 days after surgery in patients with and without migraine and migraine aura. The secondary outcome was hospital readmission within 30 days of surgery. Exploratory outcomes included post-discharge stroke and strata of neuroanatomical stroke location.

Results 10179 (8.2\%) patients had any migraine diagnosis, of whom 1278 (12.6\%) had migraine with aura and 8901 (87.4\%) had migraine without aura. $771(0.6 \%)$ perioperative ischemic strokes occurred within 30 days of surgery. Patients with migraine were at increased risk of perioperative ischemic stroke (adjusted odds ratio 1.75, 95\% confidence interval 1.39 to 2.21) compared with patients without migraine. The risk was higher in patients with migraine with aura (adjusted odds ratio 2.61, 1.59 to 4.29$)$ than in those with migraine without aura $(1.62,1.26$ to 2.09). The predicted absolute risk is 2.4 (2.1 to 2.8) perioperative ischemic strokes for every 1000 surgical patients. This increases to 4.3 (3.2 to 5.3) for every 1000 patients with any migraine diagnosis, 3.9 (2.9 to 5.0 ) for migraine without aura, and 6.3 (3.2 to 9.5) for migraine with
\end{abstract}

aura.Patients with migraine had a higher rate of readmission to hospital within 30 days of discharge (adjusted odds ratio $1.31,1.22$ to 1.41).

Conclusions Surgical patients with a history of migraine are at increased risk of perioperative ischemic stroke and have an increased 30 day hospital readmission rate. Migraine should be considered in the risk assessment for perioperative ischemic stroke.

\section{Introduction}

Up to one fifth of the general population have migraine, a primary, chronic-intermittent headache disorder affecting the neuronal and vascular systems and characterized by severe headache accompanied by nausea and/or sensory hypersensitivities such as photophobia and phonophobia. In approximately $20-30 \%$ of patients, the headache phase is preceded or accompanied by transient focal neurological disturbances presenting as visual symptoms but also sensory, aphasic, or motor symptoms known as migraine aura. ${ }^{12} \mathrm{An}$ association between a history of migraine and increased risk of ischemic stroke has been established by many observational and experimental studies. ${ }^{3-9}$

Stroke is responsible for approximately 6.2 million deaths a year and is a leading global cause of long term disability. ${ }^{10}$ Stroke within 30 days of surgery occurs in approximately 1 in 1000 surgical cases in broad surgical populations excluding cardiac, carotid, major vascular, and neurologic surgery, ${ }^{11}$ and in between $0.6 \%$ and $7.4 \%$ of patients after major cardiac and vascular surgery. ${ }^{12}{ }^{13}$ Considering that more than 50 million patients in hospital and 53 million ambulatory patients undergo surgical procedures in the United States every year, ${ }^{14}{ }^{15}$ 
perioperative stroke represents a highly relevant surgical complication. Surgical stress and post-procedural inflammatory responses have been suggested as facilitating thromboembolic formation, ${ }^{16}$ as have age, vascular comorbidities, and intraoperative hypotension, ${ }^{12-18}$ resulting in an increased risk of ischemic stroke around the time of surgery. ${ }^{17-20}$ Perioperative ischemic stroke is associated with an eightfold increase in the risk of mortality and serious long term morbidities and could lead to increased hospital readmission rates. ${ }^{11}$

As associations of migraine with risk of ischemic stroke have not yet been studied in the perioperative setting, and pathophysiologic mechanisms of migraine can also interfere with stroke risk during that period, ${ }^{821}$ we hypothesized that a diagnosis of migraine would increase the risk of perioperative ischemic stroke in a large cohort of surgical patients and would lead to increased hospital readmission rates after surgery.

\section{Methods}

\section{Study population}

We examined data from all patients who had surgery under general anesthesia with mechanical ventilation between January 2007 and August 2014 and were extubated after surgery at Massachusetts General Hospital and two community hospitals in Massachusetts, USA. Data came from different sources at Partners HealthCare and Massachusetts General Hospital: the Anesthesia Information Management System, the Research Patient Data Registry, and Enterprise Performance Systems Inc. The Anesthesia Information Management System prospectively records intraoperative physiologic data such as intraoperatively administered drug doses, intraoperative blood pressure, fluid resuscitation volumes, and blood product transfusion. We obtained demographic data and billing codes from the Research Patient Data Registry, a centralized registry that compiles electronic health record data from various institutional systems of Partners HealthCare specifically for research purposes. We collected work relative value units, a marker for cost of hospital stay and procedural complexity, ${ }^{22}$ for each surgical operation through Enterprise Performance Inc, a performance improvement and financial planning system at our institution. We used patient data from all sources to form one de-identified database, as previously reported by our group. ${ }^{23-25}$

\section{Patient involvement}

No patients were involved in setting the research question or the outcome measures, nor were they involved in developing plans for recruitment, design, or implementation of the study. No patients were asked advice on interpretation or writing up of results. Results of the study will be disseminated to migraine patients' organizations.

\section{Migraine status}

We determined history of migraine and migraine aura status on the basis of ICD-9 (international classification of diseases, ninth edition) diagnosis codes (supplementary table A). We considered patients without a billing diagnosis for migraine not to have a history of migraine.

\section{Outcome measures}

The primary outcome was perioperative ischemic stroke within 30 days after surgery. We identified patients who were billed for any ICD-9 diagnoses of ischemic stroke (supplementary table A) within 30 days of surgery. We reviewed medical records for all perioperative ischemic strokes on the basis of brain scan reports (magnetic resonance imaging/computed tomography), acute stroke and neurology consultation notes, and discharge summaries. We classified strokes on the basis of radiology reports according to the Oxfordshire Community Stroke Project classification..$^{26}$ The acquisition of the stroke date allowed us to categorize strokes into pre-discharge or post-discharge events of the initial hospital stay.

The secondary outcome was hospital readmission within 30 days of discharge, defined as an in-hospital admission to the tertiary care facility or any other affiliated hospital. We categorized readmission diagnoses according to the standardized categories of the International Statistical Classification of Diseases and Related Health Problems and stratified them by migraine status.

\section{Statistical analysis}

We used multivariable adjusted logistic regression to estimate odds ratios and $95 \%$ confidence intervals of perioperative stroke and 30 day hospital readmission associated with migraine. We evaluated the associations of migraine status and our primary and secondary outcomes by including an indicator variable contrasting migraine history versus no migraine history. In a separate multivariable logistic regression model, we further included a factor variable subdivided for migraine by aura status (no migraine, migraine without aura, migraine with aura) to consider associations between outcomes and migraine aura status. All multivariable models controlled for a priori selected potential confounding variables: sex; age $(<45,45-65,>65$ years); body mass index (<18.5, 18.5-25, 25-30, 30-35, $>35)$; American Society of Anesthesiologists physical status classification ( $<3, \geq 3)$; emergent versus non-emergent surgery; inpatient surgery; high risk surgery; Charlson Comorbidity Index (0, 1-2, 3, 4-7, >7); prescription of any antiplatelet drug or $\beta$ blockers within four weeks before surgery; history of coronary artery disease, dyslipidemia, diabetes, hypertension, atrial fibrillation, or ICD-9 codes (see supplementary table A) indicating right-to-left shunt; duration of surgery; intraoperative hypotensive minutes $(<11,11-20,>20)$; intraoperative vasopressor dose $(0,<0.05,0.05-0.25,>0.25 \mathrm{mg}$ norepinephrine (noradrenaline) equivalent ${ }^{27}$ ); intraoperative fluid volume (defined as the sum of crystalloids and colloids); requirement for blood transfusion; and work relative value units $(<7.5$, 7.5-12, 12-18, 18-25, >25).

We investigated associations between migraine and perioperative ischemic stroke location by using an age adjusted multinomial logistic regression model with perioperative stroke as outcome. We tested the risk of the perioperative ischemic stroke occurring after discharge from the initial hospital stay in perioperative ischemic stroke patients with migraine compared with perioperative ischemic stroke patients without migraine.

\section{Sensitivity analyses}

We examined the robustness of our results in several a priori planned sensitivity analyses. Firstly, we additionally adjusted the analyses for a history of transient ischemic attack to account for conditions that could clinically mimic migraine aura (see supplementary appendix).

Next, we examined differential associations of the migraine and perioperative ischemic stroke across a patient's migraine independent baseline risk of perioperative ischemic stroke. Using variables in our dataset selected on the basis of their theoretical relation with stroke risk, we estimated a patient's probability of perioperative ischemic stroke by using the following covariates: sex; age; body mass index; American 
Society of Anesthesiologists physical status classification; emergent versus non-emergent surgery; inpatient surgery; high risk surgery; Charlson Comorbidity Index; prescription of any antiplatelet drug or $\beta$ blockers within four weeks before surgery; history of coronary artery disease, dyslipidemia, diabetes, hypertension, atrial fibrillation, or possible right-to-left shunt; surgery duration; intraoperative hypotension; intraoperative vasopressor dose; intraoperative fluid volume; blood transfusion requirement; and work relative value units to account for procedural severity. We then subdivided the study population into three equal sized groups according to this empiric "background" probability score for perioperative ischemic stroke risk (low, intermediate, high risk). Next, we re-estimated the migraine-perioperative ischemic stroke association in these subgroups and calculated the $\mathrm{P}$ value for trend across these subgroups to examine heterogeneity. The intention of the "background" stroke probability score was to evaluate whether the migraine-perioperative ischemic stroke association differs according to the underlying risk of perioperative ischemic stroke, as suggested by previous studies. ${ }^{3-28}$ We evaluated whether age $(<45,45-65,>65)$, sex, and possible right-to-left shunt modified the association between migraine and perioperative ischemic stroke by including interaction terms (for example, migraine*sex) in the multivariable model.

For the primary analysis, we used the complete case method. As a sensitivity measure, we repeated the analysis using multiple imputations by chained equations. Missing variables were imputed using all variables included in the multivariable model.

\section{Exploratory analyses}

We did several post hoc exploratory analyses to further examine the associations that were discovered during the primary analysis. To clarify the reasons for readmission in our study population, we stratified the categories of ICD-9 readmission diagnoses by migraine status and compared them by using the $\chi^{2}$ test. Our analyses were further stratified and tested the migraine-perioperative ischemic stroke association for effect modification by inpatient versus ambulatory surgery. On the basis of the results of subgroup analyses in which we aimed to identify potentially meaningful risk factors for perioperative ischemic stroke in patients with migraine (see supplementary appendix), we continued to examine effect modifiers on the migraine-perioperative ischemic stroke association in the entire cohort of patients with and without migraine. Specifically, we considered whether the risks from each predictor were additive or whether the risks from the combination of factors were greater than the sum of each alone. We examined the interactions of migraine and possible right-to-left shunt, as well as migraine and intraoperative high dose vasopressor administration $(>0.25$ $\mathrm{mg}$ norepinephrine equivalent dose) on risk of perioperative ischemic stroke. To examine these interactions, we considered both absolute risk differences and relative risk as suggested by the STROBE guidelines. ${ }^{29} \mathrm{We}$ calculated the departure from additivity by using relative excess risk and confidence intervals defined by Hosmer and Lemeshow. ${ }^{30}$ We did other sensitivity and exploratory analyses, which are described in the supplementary appendix.

We used Stata version 13 for all analyses. We considered a two tailed $\mathrm{P}$ value of less than 0.05 to be statistically significant.

\section{Results}

\section{Study population}

A total of 124558 surgical cases met our inclusion criteria for analysis (fig $1 \Downarrow$ ). We identified $10179(8.2 \%)$ patients with a migraine diagnosis on the basis of ICD-9 codes, of whom 1278 $(12.6 \%)$ had migraine with aura. Patients diagnosed as having migraine, compared with those without, were more often female, were younger, and had an overall lower frequency of vascular risk factors, including diabetes, hypertension, coronary artery disease, and atrial fibrillation (table $1 \Downarrow$ ).

\section{Primary and secondary outcomes}

Among the 124558 surgical cases, 771 (0.6\%) perioperative ischemic strokes occurred. Vascular surgery had the highest rate of perioperative ischemic strokes $(244 ; 4.0 \%)$, followed by cardiac $(25 ; 3.5 \%)$ and neurosurgical procedures $(244 ; 2.4 \%)$. Of all patients with perioperative ischemic stroke, $89(11.5 \%)$ had a diagnostic code for migraine; of these, 18 (2.3\%) had migraine with aura and $71(9.2 \%)$ had migraine without aura. Patients with migraine had an increased risk for perioperative ischemic stroke compared with patients without migraine (adjusted odds ratio $1.75,95 \%$ confidence interval 1.39 to 2.21 ) (table $2 \Downarrow$ ). The risk of ischemic stroke was higher for patients with migraine with aura (adjusted odds ratio 2.61, 1.59 to 4.29) but was also apparent for migraine without aura $(1.62,1.26$ to 2.09), compared with surgical patients without migraine.

With regard to absolute risks, we predict that 2.4 (95\% confidence interval 2.1 to 2.8 ) ischemic strokes would be seen for every 1000 surgical patients. This risk increases to 4.3 (3.2 to 5.3) for every 1000 patients with any migraine diagnosis. Stratified by migraine with aura status, these numbers are 3.9 (2.9 to 5.0) for migraine without aura and 6.3 (3.2 to 9.5) for migraine with aura.

In total, 10088 patients in our study sample were readmitted to hospital within 30 days. The 30 day hospital readmission rate was higher for patients with migraine than for those with no migraine (adjusted odds ratio 1.31 1.22 to 1.41). Both migraine with aura (adjusted odds ratio 1.59, 1.33 to 1.91) and migraine without aura $(1.27,1.18$ to 1.38$)$ were associated with a higher risk of 30 day hospital readmission compared with patients without migraine.

Table $2 \Downarrow$ shows strata of stroke location based on the Oxfordshire Community Stroke Project Classification. In a multinomial analysis, the risks of perioperative posterior circulation stroke (age adjusted odds ratio 1.96, 1.26 to 3.05) and perioperative partial anterior stroke (1.95, 1.08 to 3.52$)$ were significantly increased in patients with migraine, compared with those without migraine.

Perioperative ischemic stroke in our study cohort occurred most frequently within the first two days after surgery $(356 / 771$; 46.2\%); 195/771 (25.3\%) perioperative ischemic strokes occurred after discharge from the hospital. Patients with migraine had significantly higher odds for post-discharge ischemic stroke than patients without migraine (age adjusted odds ratio $1.94,1.18$ to 3.20 ).

\section{Sensitivity analyses}

Our findings remained stable when we additionally adjusted for history of transient ischemic attack (see supplementary appendix).

We built a probability score for baseline risk of perioperative ischemic stroke, and the area under the curve for perioperative ischemic stroke was 0.85 . Clinical characteristics of subgroups by low, intermediate, and high stroke risk probability are shown in supplementary table B. Subgroup analyses in thirds of this estimated "background" ischemic stroke risk probability score showed the highest risk for the migraine-ischemic stroke 
association in patients with the otherwise lowest baseline risk of perioperative ischemic stroke. The adjusted odds ratios for the migraine-perioperative ischemic stroke association in patients with high, intermediate, and low predicted stroke risk were 1.63 (1.28 to 2.08$), 1.62$ (0.83 to 3.16), and 3.52 (1.28 to 9.7), respectively (P for trend $<0.001$ ).

Age, sex, or the presence of a possible right-to-left shunt did not modify the relative risk estimate of migraine on perioperative ischemic stroke (all P for interaction $>0.42$ ). Imputation of missing data did not affect the results of our study: the adjusted odds ratios for patients with migraine were 1.69 (1.35 to 2.11 ) for perioperative ischemic stroke and 1.33 (1.24 to 1.43) for hospital readmission.

\section{Exploratory analyses}

Readmission diagnoses were available for 9793 (97.1\%) patients who were readmitted to hospital within 30 days. Patients with migraine had statistically significantly higher frequencies of readmission due to neurological diseases $(\mathrm{P}<0.001)$, diseases of the circulatory system $(\mathrm{P}=0.002$; also in the subgroup "cerebrovascular disease", $\mathrm{P}=0.005)$, diseases of the digestive system $(\mathrm{P}=0.04)$, and "signs, symptoms, and ill defined conditions" such as pain, syncope/collapse, or nausea/vomiting $(\mathrm{P}<0.001)$ (table $3 \Downarrow)$.

The risk of perioperative ischemic stroke for migraine versus no migraine was numerically higher in the subgroup of ambulant surgical patients (adjusted odds ratio 4.02, 1.40 to 13.93) than in the subgroup of inpatient surgical patients (1.69, 1.33 to 2.15). However, the migraine-perioperative ischemic stroke association was not significantly modified by ambulatory versus inpatient surgery $(\mathrm{P}$ for interaction $=0.29$ ).

In patients with migraine, intraoperative administration of high dose vasopressor $(>0.25 \mathrm{mg})$ and ICD-9 codes indicating a possible right-to-left shunt were independent and possibly preventable predictors of perioperative stroke (see supplementary appendix). In formal further exploratory interaction analyses, the independent adjusted perioperative stroke risk for migraine with aura (6.2/1000 patients) and high dose vasopressor (3/1000 patients) combined to produce a joint risk that was numerically greater than the sum of both (11.4/1000 patients). This non-additivity resulted in a relative excess risk of 2.3 (95\% confidence interval -1.3 to 5.9 ).

Similarly, the independent adjusted risks of migraine with aura (6.2/1000) and possible right-to-left shunt (7.5/1000) combined to produce a joint risk that was greater than the sum of both (20.4/1000). This non-additivity resulted in a relative excess risk of 4.1 ( -5.1 to 13.2$)$. Neither interaction was statistically significant in this sample ( $\mathrm{P}$ for interaction $=0.77$ for possible right-to-left shunt and 0.09 for high dose vasopressors), given that the wide $95 \%$ confidence intervals could not rule out additivity, but the point estimates suggest that further investigation is warranted.

\section{Discussion}

In this large cohort of surgical patients, an ICD-9 code for migraine was associated with an increased risk of perioperative ischemic stroke within 30 days of surgery and an increased 30 day hospital readmission rate. The observed association remained consistent after adjustment for a large number of disease and surgery related risk factors for ischemic stroke. The migraine associated risk of perioperative ischemic stroke was highest in patients with migraine with aura, ambulatory patients, and patients with migraine with an otherwise low a priori estimated risk of ischemic stroke based on preoperative and intraoperative predictors of perioperative ischemic stroke.

\section{Strength and weaknesses of study}

The strengths of our study include the large number of surgical patients and patients with migraine, as well as the multi-centric design and diverse population, which allow for application of our results in a diverse range of settings. We validated occurrence of perioperative ischemic stroke by medical chart review, which allowed us to gather additional information such as location of ischemic stroke and timing of the stroke event. Moreover, we had information on a large number of potential confounding factors for which we controlled in multivariable models.

Several limitations have to be considered when interpreting our results. Firstly, our classification of migraine and migraine aura was based on ICD-9 codes using the Partners Healthcare Research Patients Data Registry dataset, which may lead to misclassification. ${ }^{31}$ ICD-9 codes are perhaps the most commonly used classification scheme in perioperative epidemiologic research. The generation of these codes is undoubtedly susceptible to error at several different points along the path from admission of the patient to inclusion into a database, but the level of accuracy may be less important than the pattern of error. If random or non-differential misclassification exists, it is unlikely to cause the observed association. ${ }^{32}$ In addition, the ICD-9 codes for migraine reported in our study correspond to reported data on prevalence of migraine in the general population, ${ }^{1}$ which is a reassuring finding. Although we made efforts to account for potential misclassification of headache type in our study (see supplementary appendix), doctors may not always record a diagnosis of migraine for mild cases or may incorrectly label them as a different type of headache. The available ICD-9 codes for migraine and migraine aura could capture a subgroup of patients with migraine with the most active, bothersome aura, which may represent a subgroup at higher risk for perioperative ischemic stroke.

\section{Comparison with other studies}

The association between migraine and ischemic stroke is well established by many clinic based and population based studies, as well as their meta-analyses..$^{3-33}$ Migraine approximately doubles the relative risk of ischemic stroke, particularly in women with migraine with aura, independent of other vascular risk factors. ${ }^{63}$ In this study, we report an important, new association between migraine and stroke occurring within 30 days after surgery, indicating that the increased risk of ischemic stroke in patients with migraine seems to exist in the very narrow perioperative time window. The perioperative ischemic stroke risk was higher in patients with migraine with aura than in those with migraine without aura, supporting most previous studies on non-perioperative migraine-ischemic stroke associations. ${ }^{633}$ Our findings were robust when we adjusted for transient ischemic attacks that can be clinically misinterpreted as migraine aura (see supplementary appendix). Population based studies have linked migraine with aura with an increased prevalence of subclinical brain infarcts and white matter hyperintensities, ${ }^{34-38}$ which themselves have been associated with increased risk of stroke, dementia, and mortality. ${ }^{39}$ In contrast, a cross sectional population based study using the Danish Twin Registry and an observational study using the Helsinki Young Stroke Registry did not find evidence of an association between silent brain infarcts, white matter hyperintensities, and migraine. ${ }^{40} 41$ These previous population based studies have identified an increased risk of ischemic stroke in patients with migraine in the context 
of stroke events occurring during many years of follow-up, whereas our study isolates this risk during the much narrower time frame of the perioperative setting.

Previous reports suggest that the migraine-ischemic stroke association is highest or only observable among patients with low vascular risk status (such as low Framingham scores or absence of hypertension, diabetes, or myocardial infarction). ${ }^{3-28}$ To consider this matter in an exploratory analysis, we needed an estimate of stroke risk independent of migraine status. Thus, we specified and estimated a group of predictors, based on theory, that could index this risk. The probability of migraine independent perioperative stroke risk was balanced by covariates that were chosen on the basis of previously identified associations with perioperative ischemic stroke and vascular risk. ${ }^{11-46}$ Our rationale was to evaluate whether the association between migraine and perioperative ischemic stroke is modified by a patient's migraine independent "background" perioperative stroke risk. Using this stroke risk model, we found that the migraine associated perioperative ischemic stroke risk was highest among patients with a low estimated probability of perioperative ischemic stroke.

Some studies report more unfavorable vascular risk profiles in patients with a history of migraine. ${ }^{74}$ However, the migraine-ischemic stroke association in our study and in other studies has been shown to be independent of vascular risk factors. ${ }^{63}$ Previous studies also suggest that the association between migraine and ischemic stroke is stronger in younger women. ${ }^{33}{ }^{48}$ In contrast, perioperative stroke rates have been shown to increase greatly with patients' age.$^{17}$ In our study, we did not find differences by migraine and sex or age. Whether this points to a different biological mechanism linking migraine and stroke in the perioperative setting should be investigated in future studies.

The overall incidence of perioperative ischemic stroke in our study was $0.6 \%$. Incidences of and risk factors for perioperative ischemic stroke in surgical patients vary according to the type of surgery, with rates up to $7.4 \%$ after cardiac and vascular procedures. ${ }^{12}{ }^{17}$ In our study, the highest rate of perioperative strokes occurred after vascular surgery $(4.0 \%)$, followed by cardiac $(3.5 \%)$ and neurosurgical $(2.4 \%)$ procedures, which are considered to be high risk surgical procedures for perioperative thromboembolic complications. ${ }^{49}$ Previously established risk factors for perioperative stroke across various surgical populations include a history of (cerebro)vascular, cardiac, and renal disease; presence of atrial fibrillation, diabetes, and hypertension; previous cardiac surgery; emergency procedure; surgical procedure type; and high transfusion requirements. ${ }^{12-17}$ We aimed to adjust our findings for these potential confounders by including these risk factors, as well as high risk procedures, in our analysis. According to our data, patients with migraine had higher frequency counts for cardiac surgery, general surgical, neurosurgical, and plastic surgery procedures, compared with patients without migraine. However, the type of surgery did not modify the risk of perioperative ischemic stroke in patients with migraine (see supplementary appendix).

Several large scale studies have shown that perioperative stroke is associated with prolonged hospital stay, long term disability, and a compelling increase in postoperative mortality risk..$^{11}$ Our data suggest that migraine represents an important risk marker for perioperative ischemic stroke among patients with a relatively low risk of ischemic stroke. The migraine associated risk of perioperative ischemic stroke was elevated in a subgroup of ambulant surgical patients (adjusted odds ratio 4.02, 95\% confidence interval 1.40 to 13.93). Particularly in times of increasing rates of ambulatory surgery, which have recently even exceeded annual rates of inpatient surgery, ${ }^{15}$ this finding of a magnified perioperative ischemic stroke risk in ambulatory surgical patients with migraine becomes highly relevant. However, inpatient versus ambulatory surgery did not statistically significantly modify the migraine-perioperative ischemic stroke association.

Only a few studies have investigated the timing of perioperative stroke after surgery in general, and they report conflicting data. ${ }^{12-51}$ In our study, whereas most strokes occurred within the first two days after surgery, the odds of a post-discharge ischemic stroke were approximately twofold higher for patients with migraine with perioperative ischemic stroke than for patients without migraine and perioperative ischemic stroke (odds ratio 1.94, 1.18 to 3.20). Migraine was also associated with an increased risk of hospital readmission within 30 days of surgery. Migraine is known to be associated with a variety of comorbidities, which are mainly cardiovascular, cerebrovascular, and neurologic. In our study, patients with migraine were more likely to be readmitted not only owing to perioperative stroke, neurologic symptoms, or other diseases of the circulatory system, but also for "signs, symptoms, and ill-defined conditions," such as pain, syncope/collapse, or nausea/vomiting and diseases of the digestive system. This is in accordance with previous reports, in which migraine has been associated with gastrointestinal disease. ${ }^{52}$ Generalized pain symptoms, as well as nausea, could be a sign of postoperative exacerbation of migraine. General practitioners must be aware of increased risks of postoperative readmission to hospital in patients with migraine, not only because of known associations such as vascular comorbidities but also due to gastrointestinal disease and circulatory problems.

Data on associations between migraine and subtypes of ischemic stroke show higher frequencies of lacunar strokes and strokes of undetermined cause in patients with migraine with aura. ${ }^{45}$ The Cerebral Abnormalities in Migraine, an Epidemiological Risk Analysis (CAMERA) study found an increased risk for subclinical cerebellar infarct-like lesions involving the posterior circulation territory in patients with migraine. ${ }^{34}$ Similarly, in our study, a history of migraine was associated with an increased risk of perioperative posterior circulation infarcts but was also observable for partial anterior infarcts.

\section{Potential biological mechanisms}

We speculate that a genetic predisposition, ${ }^{53}$ as well as an increased perioperative vulnerability to cerebral ischemia, may lead to exacerbated perioperative stroke risk in patients with migraine. Increased susceptibility to spreading depolarization, a neuroglial wave of depolarization, and the likely biologic correlate of migraine aura, ${ }^{25}$ seems to represent part of the mechanism(s) for the increased stroke risk in patients with migraine. ${ }^{9-54}$ Spreading depolarization can be induced by ischemia and induces prolonged vasoconstriction leading to decreased tissue oxygenation during migraine aura and stroke. Paradoxical embolization through pulmonary or intracardiac shunts, such as a patent foramen ovale, ${ }^{55}$ has been postulated to serve as an additional potential source of microemboli to induce cerebral ischemia and result in cryptogenic stroke..$^{57} 58$ Other factors that increase the likelihood of spreading depolarization in the perioperative phase include endothelial dysfunction and a hypercoagulable state ${ }^{59}{ }^{60}$ Impairment of endothelial function could be augmented in the perioperative state by surgical trauma associated inflammatory responses, leading to an impairment of microvascular homeostasis. General anesthesia, dehydration, and perioperative withholding of 
anticoagulation therapy may aggravate surgery induced hypercoagulability and further facilitate perioperative thrombus formation in patients with migraine, resulting in ischemia and induction of spreading depolarization in migraine susceptible brains.

Future studies should focus on targeted investigation of these interactions and potential biologic mechanisms to provide urgently needed concepts of primary and secondary, as well as perioperative, stroke prevention in patients with migraine.

\section{Implications of findings}

The results of our study add to the emerging evidence that migraine, particularly migraine with aura, should be considered a marker for increased risk of ischemic stroke, and our data extend this association to the perioperative setting. We contend that this association has clinical relevance in a growing list of risk factors that should be considered to promote disability-free survival. Furthermore, understanding this risk period offers unique opportunities to study ischemic stroke in migraine and might result in treatment considerations for patients at risk who are not undergoing surgical intervention.

Migraine headaches are often viewed as a benign condition, despite the underlying increased risk of stroke, and so far have not been in the focus of the perioperative risk assessment. Given the high prevalence of migraine in the general population, the migraine-perioperative ischemic stroke association carries public health importance, and physicians should be aware of this increased perioperative risk, particularly in patients with migraine who present without traditional risk factors for stroke. We believe that an individual perioperative risk assessment for perioperative ischemic stroke in patients with migraine undergoing surgery may be crucial.

On the basis of the results of exploratory data analysis, we speculate that the use of high dose vasopressors during surgery as well a history of a possible right-to-left shunt may represent modifiable risk factors for perioperative ischemic stroke in patients with migraine with aura. The use of high dose vasopressors during surgery may be an indicator of suboptimal preload and possibly decreased cardiac output, which can further lead to an impairment of cerebral oxygenation despite normalization of mean arterial blood pressure. ${ }^{61}{ }^{62}$ Meticulous optimization of fluid resuscitation volume during anesthesia and surgery should help to avoid excessive use of vasopressors and its associated increased vulnerability to perioperative stroke. Published data on the association between patent foramen ovale and cryptogenic ischemic stroke or transient ischemic attack are equivocal. ${ }^{63-65}$ In our study, the combination of a history of a possible right-to-left shunt and migraine with aura was associated with a substantial increase in risk for perioperative ischemic stroke, as well as a predictor for perioperative ischemic stroke in patients with migraine.

Our data also show that migraine is associated with an increased risk of 30 day hospital readmission. Readmissions strain healthcare systems with a large economic burden and serve as performance measurements of clinical care ${ }^{66}$ Almost $16 \%$ of surgical Medicare fee-for-service patients are being readmitted within 30 days after discharge, and $90 \%$ of these readmissions are estimated to be unplanned. ${ }^{66}$ Costs of unplanned hospital readmissions in 2011 amounted to $\$ 42.3 \mathrm{bn}$ ( $£ 33.7 \mathrm{bn} ; € 40.1 \mathrm{bn})$ in total hospital costs, of which Medicare carried the largest share of payments (56\%; $\$ 23.7 \mathrm{bn})$, and $\$ 568 \mathrm{~m}$ in total can be attributed to readmission due to acute cerebrovascular disease. ${ }^{67}$ With the Medicare Hospital Readmissions Reduction Program and the Centers for Medicare and Medicaid Services increasing expansion of alternative healthcare delivery systems such as bundle payments, the identification of potential causes of readmission becomes more relevant. Providers are challenged to coordinate care across treatment settings and expand initiatives for more efficient recovery by patients to balance medical and financial risk. Therefore, early detection of symptoms of stroke through close postoperative monitoring of patients at high risk should be crucial to optimize the value of perioperative care in patients with migraine.

\section{Conclusions}

In this large cohort of surgical patients, migraine was associated with an increased risk of perioperative ischemic stroke within 30 days of surgery. This risk was elevated in patients with migraine with aura, in those with a low estimated baseline risk of ischemic stroke, and in those undergoing ambulatory surgery. Migraine was also associated with a higher 30 day hospital readmission rate and higher risk of perioperative ischemic stroke after initial hospital discharge. We suggest that migraine should be included in the perioperative risk assessment.

Contributors: TK, TTH, and ME designed the study. FPT and TTH contributed to the analysis and interpretation of data and to drafting the manuscript. SDG contributed to data analysis and data acquisition. ALL and JBS contributed to data acquisition. All authors revised the manuscript critically for important intellectual content. FPT and TTH contributed equally to the work. ME served as clinical research mentor to FPT. TK and ME contributed equally and directed the work. ME is the guarantor.

Funding: This study was funded by Jeff and Judy Buzen in an unrestricted grant to ME.

Competing interests: All authors have completed the ICMJE uniform disclosure form at www.icmje.org/coi_disclosure.pdf (available on request from the corresponding author) and declare: TTH has received a research grant from Merck Inc, has served as a statistical consultant for Depomed Inc, receives payment for statistical reviewing from Anesthesiology, Annals of Surgery, and Headache, and has received research grants from National Institutes of Health (NINDS, NIGMS); TK has received grants from the Else-Kröner-Fresenius-Stiftung/German Scholars Organization, the US National Institutes of Health, and the French National Research Agency, is a consultant for Amgen on a scientific project, for which the Charité - Universitätsmedizin Berlin receives research funds, has received honorariums for editorial services from The BMJ and Cephalalgia, and, as a board of trustees member of the International Headache Society, has received compensation for travel and accommodation expenses; ME has received funding for research projects from Merck, has an equity stake at Calabash Bioscience Inc, and received funding from a research grant from the Buzen Fund, established by Jeffrey Buzen and Judith Buzen of Boston, Massachusetts; no other relationships or activities that could appear to have influenced the submitted work.

Ethical approval: This project was approved by the Partners Institutional review board (protocol No P002660).

Data sharing: No additional data available.

Transparency: The lead author affirms that the manuscript is an honest, accurate, and transparent account of the study being reported; that no important aspects of the study have been omitted; and that any discrepancies from the study as planned (and, if relevant, registered) have been explained.

Smitherman TA, Burch R, Sheikh H, Loder E. The prevalence, impact, and treatment of migraine and severe headaches in the United States: a review of statistics from national surveillance studies. Headache 2013:53:427-36. doi:10.1111/head.12074 pmid:23470015. Surveillance studies. Headache 2013;53:427-36. doi:10.1111/head.12074 pmid:23470015.
Goadsby PJ, Lipton RB, Ferrari MD. Migraine--current understanding and treatment. N Engl J Med 2002;346:257-70. doi:10.1056/NEJMra010917 pmid:11807151. 


\section{What is already known on this topic}

Migraine has been identified as a risk factor for ischemic stroke

The risk of ischemic stroke is particularly increased in patients with migraine with aura

\section{What this study adds}

Patients with migraine, particularly migraine with aura, undergoing a surgical procedure are at increased risk of perioperative ischemic stroke and readmission to hospital within 30 days after discharge

Migraine should be incorporated in the assessment for risk of perioperative stroke

Possible preventable risk factors for perioperative stroke in patients with migraine have been identified and should be further analyzed in future studies

3 Henrich JB, Horwitz RI. A controlled study of ischemic stroke risk in migraine patients. J Clin Epidemiol 1989;42:773-80. doi:10.1016/0895-4356(89)90075-9 pmid:2760669.

4 Kurth T, Slomke MA, Kase CS, et al. Migraine, headache, and the risk of stroke in women: a prospective study. Neurology 2005;64:1020-6. doi:10.1212/01.WNL.0000154528.21485 3A pmid: 15781820

5 MacClellan LR, Giles W, Cole J, et al. Probable migraine with visual aura and risk of ischemic stroke: the stroke prevention in young women study. Stroke 2007;38:2438-45. doi:10.1161/STROKEAHA.107.488395 pmid: 17690308

6 Spector JT, Kahn SR, Jones MR, Jayakumar M, Dalal D, Nazarian S. Migraine headache and ischemic stroke risk: an updated meta-analysis. Am J Med 2010;123:612-24. doi:10. 1016/j.amjmed.2009.12.021 pmid:20493462.

7 Bigal ME, Kurth T, Santanello N, et al. Migraine and cardiovascular disease: a population-based study. Neurology 2010;74:628-35. doi:10.1212/WNL. ab013e3181d0cc8b pmid:20147658.

8 Eikermann-Haerter K, Lee JH, Yuzawa I, et al. Migraine mutations increase stroke vulnerability by facilitating ischemic depolarizations. Circulation 2012;125:335-45. doi:10 1161/CIRCULATIONAHA.111.045096 pmid:22144569.

9 Kurth T, Winter AC, Eliassen AH, et al. Migraine and risk of cardiovascular disease in women: prospective cohort study. BMJ 2016;353:i2610. doi:10.1136/bmj.i2610 pmid: 27247281.

10 Moran AE, Roth GA, Narula J, Mensah GA. 1990-2010 global cardiovascular disease atlas. Glob Heart 2014;9:3-16. doi:10.1016/j.gheart.2014.03.1220 pmid:25432106.

11 Mashour GA, Shanks AM, Kheterpal S. Perioperative stroke and associated mortality after noncardiac, nonneurologic surgery. Anesthesiology 2011;114:1289-96. doi:10.1097/ ALN.0b013e318216e7f4 pmid:21478735.

12 Bucerius J, Gummert JF, Borger MA, et al. Stroke after cardiac surgery: a risk factor analysis of 16,184 consecutive adult patients. Ann Thorac Surg 2003;75:472-8. doi:10. 1016/S0003-4975(02)04370-9 pmid:12607656.

13 Sharifpour M, Moore LE, Shanks AM, Didier TJ, Kheterpal S, Mashour GA. Incidence, predictors, and outcomes of perioperative stroke in noncarotid major vascular surgery. Anesth Analg 2013;116:424-34. doi:10.1213/ANE.0b013e31826a1a32 pmid:23115255.

14 Centers for Disease Control and Prevention. National Hospital Discharge Survey. 2010. https://www.cdc.gov/nchs/data/nhds/4procedures/2010pro4_numberproceduresex.pdf.

15 Cullen KA, Hall MJ, Golosinskiy A, Division of Health Care Statistics. Ambulatory Surgery in the United States, 2006. http://www.cdc.gov/nchs/data/nhsr/nhsr011.pdf.

16 Elkind MS. Inflammatory mechanisms of stroke. Stroke 2010;41(Suppl):S3-8. doi:10.1161/ STROKEAHA.110.594945 pmid:20876499.

17 Bateman BT, Schumacher HC, Wang S, Shaefi S, Berman MF. Perioperative acute ischemic stroke in noncardiac and nonvascular surgery: incidence, risk factors, and outcomes. Anesthesiology 2009;110:231-8.pmid:19194149.

18 Bijker JB, Persoon S, Peelen LM, et al. Intraoperative hypotension and perioperative ischemic stroke after general surgery: a nested case-control study. Anesthesiology 2012;116:658-64. doi:10.1097/ALN.0b013e3182472320 pmid:22277949.

19 Wong GY, Warner DO, Schroeder DR, et al. Risk of surgery and anesthesia for ischemic stroke. Anesthesiology 2000;92:425-32. doi:10.1097/00000542-200002000-00024 pmid: 10691229.

20 Vasivej T, Sathirapanya P, Kongkamol C. Incidence and risk factors of perioperative stroke in noncardiac, and nonaortic and its major branches surgery. J Stroke Cerebrovasc Dis 2016;25:1172-6. doi:10.1016/j.jstrokecerebrovasdis.2016.01.051 pmid:26922129.

21 Dreier JP, Reiffurth C. The stroke-migraine depolarization continuum. Neuron 2015;86:902-22. doi:10.1016/..neuron.2015.04.004 pmid:25996134.

22 Davenport DL, Henderson WG, Khuri SF, Mentzer RM Jr. Preoperative risk factors and surgical complexity are more predictive of costs than postoperative complications: a case study using the National Surgical Quality Improvement Program (NSQIP) database. Ann Surg 2005;242:463-8, discussion 468-71.pmid:16192806.

23 Ladha K, Vidal Melo MF, McLean DJ, et al. Intraoperative protective mechanical ventilation and risk of postoperative respiratory complications: hospital based registry study. BMJ 2015;351:h3646. doi:10.1136/bmj.h3646 pmid:26174419

24 de Jong MA, Ladha KS, Melo MF, et al. Differential effects of intraoperative positive end-expiratory pressure (PEEP) on respiratory outcome in major abdominal surgery versus craniotomy. Ann Surg 2016;264:362-9. doi:10.1097/SLA.0000000000001499 pmid: 26496082

25 Grabitz SD, Farhan HN, Ruscic KJ, et al. Dose-dependent protective effect of inhalational anesthetics against postoperative respiratory complications: a prospective analysis of data on file from three hospitals in New England. Crit Care Med 2016. doi:10.1097/CCM. 0000000000002015 pmid:27635768.

26 Bamford J, Sandercock P, Dennis M, Burn J, Warlow C. Classification and natural history of clinically identifiable subtypes of cerebral infarction. Lancet 1991;337:1521-6. doi:10. 1016/0140-6736(91)93206-O pmid:1675378.

27 Russell JA, Walley KR, Singer J, et al. VASST Investigators. Vasopressin versus norepinephrine infusion in patients with septic shock. N Engl J Med 2008;358:877-87. doi:10.1056/NEJMoa067373 pmid:18305265.

28 Kurth T, Schürks M, Logroscino G, Gaziano JM, Buring JE. Migraine, vascular risk, and cardiovascular events in women: prospective cohort study. BMJ 2008;337:a636. doi:10. 1136/bmj.a636 pmid:18687721.

29 Vandenbroucke JP, von Elm E, Altman DG, et al. STROBE Initiative. Strengthening the Reporting of Observational Studies in Epidemiology (STROBE): explanation and elaboration. Epidemiology 2007;18:805-35. doi:10.1097/EDE.0b013e3181577511 pmid: 18049195 .

30 Assmann SF, Hosmer DW, Lemeshow S, Mundt KA. Confidence intervals for measures of interaction. Epidemiology 1996;7:286-90. doi:10.1097/00001648-19960500000012 pmid:8728443.

31 Rizzoli P, Loder E, Joshi S. Validity of cluster headache diagnoses in an electronic health record data repository. Headache 2016;56:1132-6. doi:10.1111/head.12850 pmid: 27273524.

32 Kurth T, Gaziano JM, Cook NR, Logroscino G, Diener HC, Buring JE. Migraine and risk of cardiovascular disease in women. JAMA 2006;296:283-91. doi:10.1001/jama.296.3. 283 pmid: 16849661

33 Schürks M, Rist PM, Bigal ME, Buring JE, Lipton RB, Kurth T. Migraine and cardiovascular disease: systematic review and meta-analysis. BMJ 2009;339:b3914. doi:10.1136/bmj. b3914 pmid:19861375.

34 Kruit MC, van Buchem MA, Hofman PA, et al. Migraine as a risk factor for subclinical brain lesions. JAMA 2004;291:427-34. doi:10.1001/jama.291.4.427 pmid:14747499.

35 Scher Al, Gudmundsson LS, Sigurdsson S, et al. Migraine headache in middle age and late-life brain infarcts. JAMA 2009;301:2563-70. doi:10.1001/jama.2009.932 pmid: 19549973.

36 Kurth $\mathrm{T}$, Mohamed $\mathrm{S}$, Maillard $\mathrm{P}$, et al. Headache, migraine, and structural brain lesions and function: population based Epidemiology of Vascular Ageing-MRI study. BMJ 2011;342:c7357. doi:10.1136/bmj.c7357 pmid:21245119.

37 Palm-Meinders $\mathrm{IH}$, Koppen $\mathrm{H}$, Terwindt GM, et al. Structural brain changes in migraine. JAMA 2012;308:1889-97. doi:10.1001/jama.2012.14276 pmid:23150008.

38 Monteith T, Gardener H, Rundek T, et al. Migraine, white matter hyperintensities, and subclinical brain infarction in a diverse community: the northern Manhattan study. Stroke 2014;45:1830-2. doi:10.1161/STROKEAHA.114.005447 pmid:24876263.

39 Debette S, Markus HS. The clinical importance of white matter hyperintensities on brain magnetic resonance imaging: systematic review and meta-analysis. BMJ 2010;341:c3666. doi:10.1136/bmj.c3666 pmid:20660506.

40 Gaist D, Garde E, Blaabjerg M, et al. Migraine with aura and risk of silent brain infarcts and white matter hyperintensities: an MRI study. Brain 2016;139:2015-23. doi:10.1093/ brain/aww099 pmid:27190013.

41 Putaala J, Kurkinen M, Tarvos V, Salonen O, Kaste M, Tatlisumak T. Silent brain infarcts and leukoaraiosis in young adults with first-ever ischemic stroke. Neurology 2009;72:1823-9. doi:10.1212/WNL.0b013e3181a711df pmid:19470964.

42 Mashour GA, Sharifpour M, Freundlich RE, et al. Perioperative metoprolol and risk of stroke after noncardiac surgery. Anesthesiology 2013:119:1340-6. doi:10.1097/ALN. bb013e318295a25f pmid:23612244.

43 Macellari F, Paciaroni M, Agnelli G, Caso V. Perioperative stroke risk in nonvascular surgery. Cerebrovasc Dis 2012;34:175-81. doi:10.1159/000339982 pmid:22922182.

44 Calvillo-King L, Xuan L, Zhang S, Tuhrim S, Halm EA. Predicting risk of perioperative death and stroke after carotid endarterectomy in asymptomatic patients: derivation and validation of a clinical risk score. Stroke 2010;41:2786-94. doi:10.1161/STROKEAHA. 110.599019 pmid:21051669.

45 Stavrinou P, Bergmann J, Palkowiz S, Goldbrunner R, Rieger B. Identifying risk factors and proposing a risk-profile scoring scale for perioperative ischemic complications in carotid endarterectomies. J Neurosurg Sci 2016;60:11-7.pmid:24844171.

46 Su AW, Habermann EB, Thomsen KM, Milbrandt TA, Nassr A, Larson AN. Risk factors for 30-day unplanned readmission and major perioperative complications after spine fusion surgery in adults: a review of the National Surgical Quality Improvement Program database. Spine (Phila Pa 1976) 2016;41:1523-34. doi:10.1097/BRS. 0000000000001558 pmid:26967124.

47 Scher Al, Terwindt GM, Picavet HS, Verschuren WM, Ferrari MD, Launer LJ. Cardiovascular risk factors and migraine: the GEM population-based study. Neurology 2005:64:614-20. doi:10.1212/01.WNL.0000151857.43225.49 pmid:15728281.

48 Peng KP, Chen YT, Fuh JL, Tang CH, Wang SJ. Migraine and incidence of ischemic stroke: A nationwide population-based study. Cephalalgia 2016;0333102416642602.pmid: 27118220.

49 Selim M. Perioperative stroke. N Engl J Med 2007;356:706-13. doi:10.1056/ NEJMra062668 pmid:17301301.

50 Parikh S, Cohen JR. Perioperative stroke after general surgical procedures. N Y State $J$ Med 1993;93:162-5.pmid:8455845.

51 Likosky DS, Marrin CA, Caplan LR, et al. Northern New England Cardiovascular Disease Study Group. Determination of etiologic mechanisms of strokes secondary to coronary artery bypass graft surgery. Stroke 2003;34:2830-4. doi:10.1161/01.STR.0000098650. 12386.B3 pmid:14605327.

52 Cámara-Lemarroy CR, Rodriguez-Gutierrez R, Monreal-Robles R, Marfil-Rivera A. Gastrointestinal disorders associated with migraine: A comprehensive review. World J Gastroenterol 2016;22:8149-60. doi:10.3748/wjg.v22.i36.8149 pmid:27688656.

53 Malik R, Freilinger T, Winsvold BS, et al. International Headache Genetics Consortium METASTROKE Collaboration of the International Stroke Genetics Consortium. Shared genetic basis for migraine and ischemic stroke: A genome-wide analysis of common variants. Neurology 2015;84:2132-45. doi:10.1212/WNL.0000000000001606 pmid: 25934857.

54 Eikermann-Haerter K. Spreading depolarization may link migraine and stroke. Headache 2014;54:1146-57. doi:10.1111/head.12386 pmid:24913618. 
55 Schwedt TJ, Demaerschalk BM, Dodick DW. Patent foramen ovale and migraine: a quantitative systematic review. Cephalalgia 2008;28:531-40. doi:10.1111/j.1468-2982. 2008.01554.x pmid:18355348.

56 Anzola GP, Magoni M, Guindani M, Rozzini L, Dalla Volta G. Potential source of cerebral embolism in migraine with aura: a transcranial Doppler study. Neurology 1999;52:1622-5. doi:10.1212/WNL.52.8.1622 pmid:10331688.

57 Alsheikh-Ali AA, Thaler DE, Kent DM. Patent foramen ovale in cryptogenic stroke: incidental or pathogenic?Stroke 2009;40:2349-55. doi:10.1161/STROKEAHA.109.547828 pmid: 19443800.

58 Nozari A, Dilekoz E, Sukhotinsky I, et al. Microemboli may link spreading depression, migraine aura, and patent foramen ovale. Ann Neurol 2010;67:221-9. doi:10.1002/ana. 21871 pmid:20225282.

59 Tietjen GE. Migraine as a systemic vasculopathy. Cephalalgia 2009;29:987-96. doi:10. 1111/j.1468-2982.2009.01937.x pmid:19689607.

60 Crassard I, Conard J, Bousser MG. Migraine and haemostasis. Cephalalgia 2001;21:630-6. doi:10.1046/j.1468-2982.2001.00205.x pmid:11531894.

61 Maas JJ, Pinsky MR, de Wilde RB, de Jonge E, Jansen JR. Cardiac output response to norepinephrine in postoperative cardiac surgery patients: interpretation with venous return and cardiac function curves. Crit Care Med 2013;41:143-50. doi:10.1097/CCM. Ob013e318265ea64 pmid:23128382.

62 Brassard $\mathrm{P}$, Seifert $\mathrm{T}$, Secher $\mathrm{NH}$. Is cerebral oxygenation negatively affected by infusion of norepinephrine in healthy subjects?Br J Anaesth 2009;102:800-5. doi:10.1093/bja/ aep065 pmid:19376788.

63 Petty GW, Khandheria BK, Meissner I, et al. Population-based study of the relationship between patent foramen ovale and cerebrovascular ischemic events. Mayo Clin Proc 2006;81:602-8. doi:10.4065/81.5.602 pmid:16706256.
64 Di Tullio MR, Sacco RL, Sciacca RR, Jin Z, Homma S. Patent foramen ovale and the risk of ischemic stroke in a multiethnic population. J Am Coll Cardiol 2007;49:797-802. doi: 10.1016/j.jacc.2006.08.063 pmid:17306710.

65 Meissner I, Khandheria BK, Heit JA, et al. Patent foramen ovale: innocent or guilty? Evidence from a prospective population-based study. J Am Coll Cardiol 2006;47:440-5. doi:10.1016/j.jacc.2005.10.044 pmid:16412874.

66 Jencks SF, Williams MV, Coleman EA. Rehospitalizations among patients in the Medicare fee-for-service program. N Engl J Med 2009;360:1418-28. doi:10.1056/ NEJMsa0803563 pmid:19339721.

67 Hines Al. BML, Jiang H.J., Steiner C.A. Conditions with the largest number of adult hospital readmissions by payer, 2011. http://www.hcup-us.ahrq.gov/reports/statbriefs/sb172Conditions-Readmissions-Payer.pdf.

Accepted: 05122016

Published by the BMJ Publishing Group Limited. For permission to use (where not already granted under a licence) please go to http://group.bmj.com/group/rights-licensing/ permissions

This is an Open Access article distributed in accordance with the Creative Commons Attribution Non Commercial (CC BY-NC 3.0) license, which permits others to distribute, remix, adapt, build upon this work non-commercially, and license their derivative works on different terms, provided the original work is properly cited and the use is non-commercial. See: http://creativecommons.org/licenses/by-nc/3.0/. 


\section{Tables}

\begin{tabular}{|c|c|c|c|c|c|}
\hline \multirow[b]{2}{*}{ Characteristics } & \multirow{2}{*}{$\begin{array}{c}\text { Total study } \\
\text { population }(n=124 \\
558)\end{array}$} & \multirow[b]{2}{*}{$\begin{array}{l}\text { No migraine ( } \mathrm{n}=114 \\
379)\end{array}$} & \multicolumn{3}{|c|}{ Migraine diagnosis $(n=10179)$} \\
\hline & & & $\begin{array}{l}\text { Any migraine }(n=10 \\
179)\end{array}$ & $\begin{array}{l}\text { Migraine without aura } \\
\qquad(\mathrm{n}=8901)\end{array}$ & $\begin{array}{l}\text { Migraine with aura } \\
(n=1278)\end{array}$ \\
\hline Mean (SD) age, years & $52.6(18.3)$ & $53.1(18.4)$ & $47.4(15.9)$ & $47.7(15.9)$ & $45.1(15.2)$ \\
\hline Female sex & $67943(54.6)$ & $59800(52.3)$ & $8143(80.0)$ & $7105(79.8)$ & $1038(81.2)$ \\
\hline Mean (SD) body mass index & $28.3(7.3)$ & $28.3(7.2)$ & $28.9(8.1)$ & $29.0(8.1)$ & $28.3(7.6)$ \\
\hline $\begin{array}{l}\text { [Median (IQR)?]ASA physical status } \\
\text { classification }\end{array}$ & $2(2-3)$ & $2(2-3)$ & $2(2-3)$ & $2(2-3)$ & $2(2-3)$ \\
\hline Emergency status & $5469(4.4)$ & $5085(4.5)$ & $384(3.8)$ & $340(3.8)$ & $44(3.4)$ \\
\hline Inpatient & $94929(76.2)$ & $87299(76.3)$ & $7630(75.0)$ & $6721(75.5)$ & $909(71.1)$ \\
\hline $\begin{array}{l}\text { Median (IQR) Charlson Comorbidity } \\
\text { Index }\end{array}$ & $1(0-3)$ & $1(0-3)$ & $1(0-2)$ & $1(0-2)$ & $1(0-3)$ \\
\hline $\begin{array}{l}\text { Any prescription of antiplatelet drug } \\
\text { within } 4 \text { weeks before surgery }\end{array}$ & $14054(11.3)$ & $13214(11.6)$ & $840(8.3)$ & $730(8.2)$ & $110(8.6)$ \\
\hline $\begin{array}{l}\text { Any prescription of } \beta \text { blockers within } 4 \\
\text { weeks before surgery }\end{array}$ & $16307(13.1)$ & $15227(13.3)$ & $1080(10.6)$ & $926(10.4)$ & $154(12.1)$ \\
\hline \multicolumn{6}{|l|}{ Stroke risk factors: } \\
\hline Diabetes & $8971(7.2)$ & $8509(7.4)$ & $462(4.5)$ & $405(4.6)$ & $55(4.3)$ \\
\hline Hypertension & $51601(41.4)$ & $47764(41.8)$ & $3837(37.7)$ & $3353(37.7)$ & $484(37.9)$ \\
\hline Dyslipidemia & $39115(31.4)$ & $35897(31.4)$ & $3218(31.6)$ & $2795(31.4)$ & $423(33.1)$ \\
\hline Coronary artery disease & $8662(7.0)$ & $8077(7.1)$ & $585(5.8)$ & $494(5.5)$ & $91(7.1)$ \\
\hline Atrial fibrillation & $8088(6.5)$ & $7607(6.7)$ & $481(4.7)$ & $397(4.5)$ & $84(6.6)$ \\
\hline Possible right-to-left shunt & $1530(1.2)$ & $1325(1.2)$ & $205(2.0)$ & $155(1.7)$ & $50(3.9)$ \\
\hline History of transient ischemic attack & $1744(1.4)$ & $1419(1.2)$ & $325(3.2)$ & $227(2.6)$ & $98(7.7)$ \\
\hline \multicolumn{6}{|l|}{ Intraoperative characteristics: } \\
\hline $\begin{array}{l}\text { Median (IQR) duration of procedure, } \\
\text { hours }\end{array}$ & $2.4(1.5-3.8)$ & $2.5(1.5-3.8)$ & $2.4(1.5-3.7)$ & $2.4(1.5-3.7)$ & $2.3(1.4-3.5)$ \\
\hline $\begin{array}{l}\text { Median (IQR) intraoperative } \\
\text { hypotensive minutes < MAP } 55 \mathrm{~mm} \mathrm{Hg}\end{array}$ & $0(0-2)$ & $0(0-2)$ & $0(0-2)$ & $0(0-2)$ & $0(0-2)$ \\
\hline $\begin{array}{l}\text { Median (IQR) total intraoperative } \\
\text { norepinephrine equivalent dose, } \mathrm{mg}\end{array}$ & $0(0-0.2)$ & $0(0-0.2)$ & $0(0-0.1)$ & $0(0-0.1)$ & $0(0-0.1)$ \\
\hline $\begin{array}{l}\text { Median (IQR) total intraoperative } \\
\text { fluids, } \mathrm{mL}\end{array}$ & $1250(800-2000)$ & $1250(800-2000)$ & $1200(800-2000)$ & $1250(800-2000)$ & $1100(750-2000)$ \\
\hline $\begin{array}{l}\text { Packed red blood cell units transfused } \\
\text { intraoperatively }\end{array}$ & $4333(3.5)$ & $4112(3.6)$ & $221(2.2)$ & $198(2.2)$ & $23(1.8)$ \\
\hline Median (IQR) work relative value units & $14.5(8.2-21.9)$ & $14.5(8.1-22.0)$ & $14.2(8.8-21.2)$ & $14.2(8.9-21.8)$ & $12(7.6-18.1)$ \\
\hline
\end{tabular}

$\mathrm{ASA}=\mathrm{American}$ Society of Anesthesiologists; $\mathrm{IQR}=$ interquartile range; $M A P=$ mean arterial pressure. 
Table 2| Incidences and univariable and multivariable adjusted odds ratios for study outcomes and strata of stroke location according to migraine status. Values are numbers (percentages) unless stated otherwise

\begin{tabular}{|c|c|c|c|c|c|}
\hline \multirow[b]{2}{*}{ Outcome } & \multicolumn{3}{|c|}{ No migraine $(n=114$} & \multicolumn{2}{|c|}{ Odds ratio $(95 \% \mathrm{Cl})$} \\
\hline & Total $(n=124558)$ & 379) & Migraine ( $n=10$ 179) & Unadjusted & Adjusted \\
\hline Perioperative ischemic stroke & $771(0.6)$ & $682(0.6)$ & $89(0.9)$ & 1.47 (1.18 to 1.84 ); $P=0.001$ & 1.75 (1.39 to 2.21$) ; P<0.001$ \\
\hline \multicolumn{6}{|l|}{$\begin{array}{l}\text { Stroke subtypes according to } \\
\text { Oxfordshire Community Stroke } \\
\text { Project classification': }\end{array}$} \\
\hline Total anterior stroke & $223(0.2)$ & $205(0.2)$ & $18(0.2)$ & 0.99 (0.61 to 1.6$) ; P=0.97$ & $1.21(0.74 \text { to } 1.97)^{\ddagger} ; P=0.44$ \\
\hline Partial anterior stroke & $102(0.1)$ & $89(0.1)$ & $13(0.1)$ & 1.65 (0.92 to 2.95); $P=0.09$ & $1.95(1.08 \text { to } 3.52)^{\ddagger} ; P=0.03$ \\
\hline Posterior circulation stroke & $181(0.1)$ & $158(0.1)$ & $23(0.2)$ & 1.64 (1.06 to 2.54$) ; P=0.03$ & $1.96(1.26 \text { to } 3.05)^{\ddagger} ; P=0.003$ \\
\hline Lacunar stroke & $30(0)$ & $28(0)$ & $2(0)$ & 0.80 (0.19 to 3.38 ); $P=0.77$ & $1.13(0.27 \text { to } 4.79)^{\ddagger} ; P=0.87$ \\
\hline Unclassifiable & $235(0.2)$ & $202(0.2)$ & $33(0.3)$ & 1.84 (1.27 to 2.66 ); $P=0.001$ & $2.33(1.61 \text { to } 3.39)^{\ddagger} ; P<0.001$ \\
\hline $\begin{array}{l}\text { Median (interquartile range) } \\
\text { days until stroke }\end{array}$ & $3(1-11)$ & $3(1-10)$ & $5.5(0-14.5)$ & & \\
\hline Post-discharge stroke & $195(0.2)$ & $164(0.1)$ & $31(0.3)$ & 1.76 ( 1.08 to 2.85 ); $P=0.02$ & $1.94(1.18 \text { to } 3.20)^{\ddagger} ; P=0.009$ \\
\hline $\begin{array}{l}\text { Hospital readmission within } 30 \\
\text { days }\end{array}$ & $10088(8.1)$ & $9160(8.0)$ & $928(9.1)$ & 1.16 (1.08 to 1.24$) ; P<0.001$ & $1.31(1.22 \text { to } 1.41)^{\circ} ; P<0.001$ \\
\hline
\end{tabular}

*Adjusted for age; sex; body mass index; American Society of Anesthesiologists physical status classification; emergent $v$ non-emergent surgery; admission type (inpatient); Charlson Comorbidity Index; history of diabetes, hypertension, atrial fibrillation, dyslipidemia, possible right-to-left-shunt, coronary artery disease; any prescription of antiplatelet drug or $\beta$ blockers within 4 weeks before surgery; duration of surgery; total intraoperative fluid volume; total intraoperative vasopressor dose (norepinephrine equivalent); intraoperative packed red blood cell units received; work relative value units.

†Associations between stroke subtypes (reference: no stroke) and migraine were analyzed using a multinomial logistic regression model.

$\ddagger$ Adjusted for age. 
Table 3| ICD-9 categories of readmission diagnoses according to migraine status. Values are numbers (percentages) unless stated otherwise

\begin{tabular}{lcccc} 
Readmission diagnoses (ICD-9 codes) & Total (n=9793) & No migraine (n=8888) & Migraine (n=905) & P value \\
\hline Infectious and parasitic diseases (001-139) & $165(1.7)$ & $152(1.7)$ & $13(1.4)$ & 0.54 \\
\hline Neoplasms (140-239) & $1203(12.3)$ & $1140(12.8)$ & $63(7.0)$ & $<0.001$ \\
\hline Endocrine, nutritional, metabolic, and immunity disorders (240-279) & $313(3.2)$ & $278(3.1)$ & $35(3.9)$ & 0.23 \\
\hline Diseases of the blood and blood-forming organs (280-289) & $101(1.0)$ & $94(1.1)$ & $7(0.8)$ & 0.42 \\
\hline Mental disorders (290-319) & $135(1.4)$ & $118(1.3)$ & $17(1.9)$ & 0.18 \\
\hline Diseases of the nervous system and sense organs (320-389) & $234(2.4)$ & $197(2.2)$ & $37(4.1)$ & $<0.001$ \\
\hline Diseases of the circulatory system (390-459) & $599(6.1)$ & $565(6.4)$ & $34(3.8)$ & 0.002 \\
\hline Cerebrovascular disease (430-438) & $105(1.1)$ & $87(1.0)$ & $18(2.0)$ & 0.005 \\
\hline Diseases of the respiratory system (460-519) & $342(3.5)$ & $310(3.5)$ & $32(3.5)$ & 0.94 \\
\hline Diseases of the digestive system (520-579) & $1016(10.4)$ & $903(10.2)$ & $113(12.5)$ & 0.04 \\
\hline Diseases of the genitourinary system (580-629) & $571(5.8)$ & $517(5.8)$ & $54(6.0)$ & 0.85 \\
\hline Complications of pregnancy, childbirth, and the puerperium (630-679) & $20(0.2)$ & $14(0.2)$ & $6(0.7)$ & 0.001 \\
\hline Diseases of the skin and subcutaneous tissue (680-709) & $220(2.2)$ & $205(2.3)$ & $15(1.7)$ & 0.21 \\
\hline Diseases of the musculoskeletal system and connective tissue $(710-739)$ & $299(3.1)$ & $266(3.0)$ & $33(3.6)$ & 0.28 \\
\hline Congenital anomalies (740-759) & $56(0.6)$ & $48(0.5)$ & $8(0.9)$ & 0.19 \\
\hline Symptoms, signs, and ill-defined conditions (780-799) & $2027(20.7)$ & $1796(20.2)$ & $231(25.5)$ & $<0.001$ \\
\hline Injury and poisoning (800-999) & $2177(22.2)$ & $1997(22.5)$ & $180(19.9)$ & 0.08 \\
\hline Other & $210(2.1)$ & $201(2.3)$ & $9(1.0)$ & 0.02 \\
\hline
\end{tabular}

Data are presented for 9793 (97.1\%) patients with available readmission diagnoses.

*For comparisons between categories of readmission diagnoses and migraine status using $x^{2}$ test. 


\section{Figure}

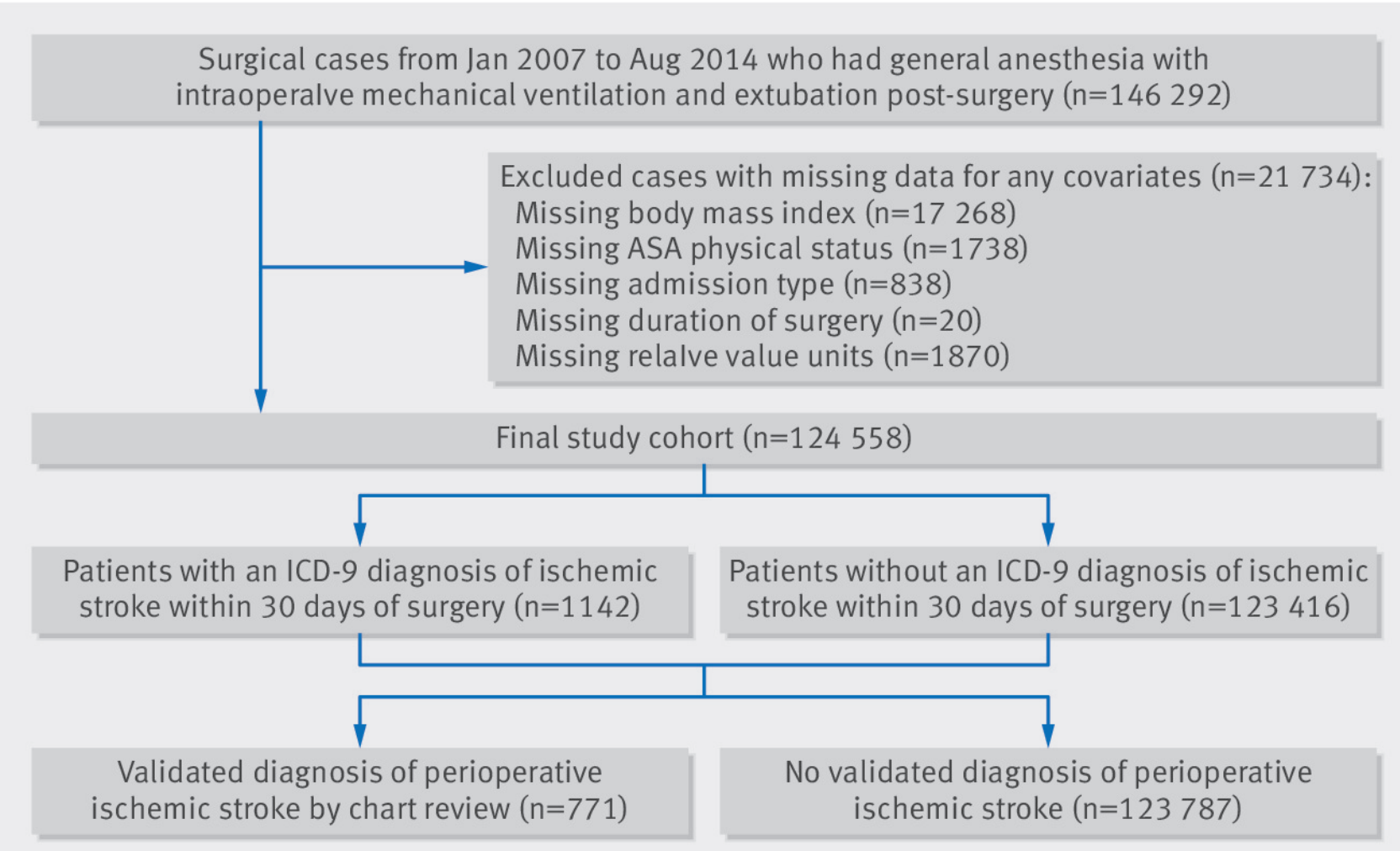

Fig 1 Flow chart of study. ASA=American Society of Anesthesiologists 